A. Fujiki

Nagoya Math. J.

Vol. 126 (1992), 89-101

\title{
REMARKS ON EXTREMAL KÄHLER METRICS ON RULED MANIFOLDS
}

\author{
AKIRA FUJIKI
}

\section{Introduction}

Let $X$ be a compact Kähler manifold and $\gamma$ a Kähler class. For a Kähler metric $g$ on $X$ we denote by $R_{g}$ the scalar curvature on $X$ According to Calabi [3][4], consider the functional $\Phi(g)=\int R_{g}^{2} d v_{g}$ defined on the set of all the Kähler metrics $g$ whose Kähler forms belong to $\gamma$, where $d v_{g}$ is the volume form associated to $g$. Such a Kähler metric is called extremal if it gives a critical point of $\Phi$. In particular, if $R_{\boldsymbol{g}}$ is constant, $\boldsymbol{g}$ is extremal. The converse is also true if $\operatorname{dim} L(X)=0$, where $L(X)$ is the maximal connected linear algebraic subgroup of Aut $_{0} X$ (cf. [5]). Note also that any Kähler-Einstein metric is of constant scalar curvature.

As for the existence of Kähler metrics with $\boldsymbol{R}_{\boldsymbol{g}}$ constant and also of extremal Kähler metrics some necessary conditions have been obtained in [15] [4](cf. Lemma 9 below) and [9]. Accordingly, there exist many examples of compact Kähler manifolds which admit no such metrics (cf. [14] and Remark 2 below). Though the above conditions become trivial in the case $L(X)=\{e\}$, Burns and de Baltolomeis [1] found examples of ruled surfaces $X$ with a Kähler class $\gamma$ such that $L(X)=$ Aut $_{0} X=\{e\}$ and $\gamma$ contains no extremal Kähler metric. On the other hand, as for the uniqueness, nothing seems to be known for the Kähler-Einstein case (cf: [2] [22]).

Note also that this problem of uniqueness and existence is important from the moduli point of view; in [8] we have constructed the moduli space of extremal Kähler metrics, and the uniqueness and existence correspond respectively to the injectivity and surjectivity of the natural map of this space into the corresponding moduli space of compact Kähler manifolds. It would therefore be of interest to study the problem even in a very special case. In this note we study the case of ruled manifolds over a compact Riemann surface and gather some remarks for this

Received January 25, 1989. 
case.

The paper is arranged as follows. Section 1 is preliminary. We determine in Section 2 the Kähler classes on ruled manifolds, and then in Section 3 consider generalized Kähler Einstein metrics. In Section 4 we treat the special case where either the base curve is an elliptic curve or the manifold comes from a finite representation of the fundamental group of the base curve. In Section 5 we supplement the above result of Burns and de Baltolomeis by determining ruled surfaces of vanishing scalar curvature. In Section 6 we discuss an interesting relation between existence and uniqueness problems.

This work was done during the author's stay at SFB 170 in 1988; he would like to thank Prof. H. Grauert and SFB 170 for the hospitality, and also to thank Prof. M. Maruyama for discussions about the subject of this paper.

\section{§ 1. Preliminaries}

The notations of this section will be used throughout this note. We fix a compact connected Riemann surface $C$ and a positive integer $r$. Denote by $\mathbf{P}$ the complex projective space of dimension $r-1$. A ruled manifold is by definition a holomorphic P-bundle over $C$, which is then written in the form $X=\mathbf{P}(E)$ for some (holomorphic) vector bundle $E$, where $\mathbf{P}(E)=(E-C) / \mathbf{C}^{*}$ with $C$ identified with the zero section.

We denote by $\ell$ and $f$ the classes in $H^{2}(X, \mathbf{R})$ of the (negative) tautological line bundle $L=L(E)$ of $E$, and of a fiber of the projection $q: X \rightarrow \mathbf{C}$ respectively. We also set

$$
e=c_{1}(X / S) / r=-c_{1}\left(K_{X / S}\right) / \gamma \in H^{2}(X, \mathbf{R}) .
$$

From the relative canonical bundle formula $K_{X / C}=L^{r} \otimes q^{*}$ det $E^{*}$ for the projection $q: X \rightarrow C$ we get

$$
e=-\ell+\mu(E) f,
$$

where $\mu(E)=\operatorname{deg} E / \operatorname{rank} E$ as usual. The various intersection numbers are given by:

$$
e^{r}=0,(-\ell)^{r}=-d, e^{r-1} \cdot f=1, e^{r-j} \cdot f^{j}=0, j \geq 2,
$$

where $d=\operatorname{deg} E$

Let $h$ be a hermitian metric on $E$ with curvature form $\Theta$, which is an End $E$-valued $(1,1)$-form on $X$. This induces a natural hermitian metric on $L$ whose curvature form $\Theta_{L}$ is described as a difference of two $(1,1)$-forms on $X$ as follows (cf. $[10 ; \S 2(\mathrm{~g})])$; 


$$
(i / 2 \pi) \Theta_{L}=-\omega_{X / C}+(i / 2 \pi) \bar{\Theta}
$$

where for any $\bar{\xi} \in X$,

$$
\bar{\Theta}_{\bar{\xi}}:=h(\Theta \xi, \xi) / h(\xi, \xi)
$$

with $\xi$ any representative of $\bar{\xi}$ in $E-C$; furthermore, $\omega_{X / C}$ has the property that 1) it is a Kähler form on each fiber and 2) if $T_{X}=T_{X / C} \oplus H$ is the $C^{\infty}$ splitting of the holomorphic tangent bundle $T_{X}$ into the vertical and a horizontal components via the hermitian connection of $(E, h)$, then any horizontal vectors annihilate $\omega_{X / C}$, where $T_{X / C}$ is the relative tangent bundle.

Fix a Kähler-Einstein form $\omega_{C}$ with total volume one on $C$, which is unique if the genus $g=g(C) \geq 2$. A hermitian vector bundle is called Hermitian-Einstein (with respect to $\omega_{C}$ ) if we have the equality of the curvature forms

$$
(i / 2 \pi) \Theta=\mu(E) I_{E} \otimes \omega_{c}
$$

where $I_{E}$ is the identity of $E$. In this case we have $(i / 2 \pi) \Theta_{L}=\mu(E) q^{*} \omega_{C}$. We call $X=P(E)$ Hermitian-Einstein if so is $(E, h)$ for some $h$.

Let $\widetilde{C} \rightarrow C$ be the universal covering map and $Y=\widetilde{C} \times \mathbf{P}$. Given a projective unitary representation $\rho: \pi \rightarrow K:=P U(r)$ of the fundamental group $\pi$ of $C$ we consider the ruled manifold $X_{\rho}:=Y / \Gamma$, where $\Gamma_{\rho}=\{(\gamma, \rho(\gamma) ; \gamma \in \pi\}$ regarded as a subgroup of Aut $Y$.

Take a $K$-invariant Kähler-Einstein form $\omega_{\mathbf{P}}$ on $\mathbf{P}$ with total volume 1 . If $p: Y \rightarrow \mathbf{P}$ is the projection, $p^{*} \omega_{\mathbf{P}}$ descends to a $d$-closed $(1,1)$-form $\omega_{q}$ on $X=X_{\rho}$.

Lemma 1. $\omega_{q}$ represents the class $e$.

Proof. Take a unique volume form $v$ on $\mathbf{P}$ with total volume 1 and with its Ricci form $\xi$ satisfying $(i / 2 \pi) \xi=r \omega_{\mathbf{P}}$. Since $\xi$ is $\pi$-invariant as well as $\omega_{\mathbf{P}}, p^{*} v$ descends to a relative volume form for $q$, or equivalently, a hermitian metric $h$ on $K_{\bar{X}}^{-1} / \mathrm{s}$. Then it suffices to check that $(i / 2 \pi) \log h=r \omega_{q}$ which follows readily from the defining equation $(i / 2 \pi) \xi=r \omega_{\mathbf{P}}$ as above.

By Lemma 1 for any real numbers $a, b$

$$
\omega_{a b}:=a \omega_{a}+b_{q}{ }^{*} \omega_{C}
$$

represents the class $\gamma_{a b}=a e+b f$ on $X_{\rho}$, which is a Kähler form if $a, b>0$.

Recall that $E$ is said to be stable (resp. semistable) if $\mu(F)<\mu(E)$ (resp. $\leq$ ). The notions descend to those for $X=\mathbf{P}(E)$. We also recall the following basic result of Narasimhan and Seshadri [18] (cf. also [19]), which is true for curves of 
any genus.

LEMma 2. For a ruled manifold $X$ the following three conditions are equivalent: 1) $X$ is isomorphic to $\boldsymbol{P}(E)$, where $E$ is a direct sum of stable vector bundles $E_{i}$ with one and the same $\mu=\mu\left(E_{i}\right)$.2) $X$ is isomorphic to $X_{\rho}$ for some representation $\rho: \pi \rightarrow K$.3) $X$ is Hermitian-Einstein.

We shall call a ruled manifold satisfying the above equivalent conditions quasi-stable.

\section{§ 2. Kähler classes on ruled manifolds}

All the vector bundles will be over $C$. Let $E$ be a vector bundle and $X=$ $\mathbf{P}(E)$. We set

$$
\mu_{\max }(E)=\max \{\mu(F) ; F \varsubsetneqq E \text { subbundle }\} .
$$

Though this depends on $E$, the number

$$
t(X)=\mu_{\max }(E)-\mu(E)
$$

depends only on $X$. Then we prove

PROPOSition 1. Let $X$ be a ruled manifold. Let $\gamma=\gamma_{a b}=a e+b f$ be a class in $H^{2}(X, \mathbf{R})$. Then $\gamma$ is a Kähler class if and only if $a>0$ and $b>\max (0$, at $(X))$.

Remark 1. 1) When $\gamma \in H^{2}(X, \mathbf{Q})$, the result is known in a somewhat different formulation (cf. [13]). 2) $\max (0, a t(X))=0$ if and only if $X$ is stable.

By using the Harder-Narasimhan filtration $[11 ; 1.3]$ from the definition of stability one gets easily the following well-known:

LEMMA 3. Every vector bundle admits an increasing filtration $0=E_{0} \subseteq \cdots \subseteq$ $E_{m}=E$ of subbundles such that 1$) F_{i}:=E_{i} / E_{i-1}$ are stable for all $i \geq 1$, 2) $\mu\left(F_{1}\right) \geq \mu\left(F_{\imath}\right)$ for all $i \geq 2$, and 3$) \mu\left(F_{1}\right)=\mu_{\max }$.

If we set $F:=\bigoplus_{i=1}^{m} F_{i}$ in the above lemma with the increasing filtration by $\oplus_{i=1}^{k} F_{i}, 1 \leq k \leq m$, then the lemma obviously applies also to $F$ instead of to $E$. The following lemma is also more or less standard.

Lemma 4. Let $E, 0=E_{0} \subseteq E_{1} \subseteq \cdots \subseteq E_{m}$, and $F_{2}$ be as in Lemma 3. Let $F:=\bigoplus_{i=1}^{m} F_{i}$. Then there exists a holomorphic family $\left\{E_{t}\right\}_{t \in \mathbf{C}}$ of vector bundles parametrized by the complex line $C$ such that $E_{0}=F$ and $E_{t} \cong E$ for any $t \neq 0$. 
Proof. Under our assumption the structure group of $E$ is reduced to a parabolic subgroup $P$ of $G L(r, \mathbf{C})$ fixing a certain flag $\left\{W_{i}\right\}$ of subspaces of $\mathbf{C}^{r}$, where $r=\operatorname{rank} E$; with respect to the standard basis $\left\{e_{j}\right\}_{1 \leq j \leq r}$ we may assume that $W_{i}$ is spanned by $e_{j}, 1 \leq j \leq r_{i}$, where $r_{i}=\operatorname{rank} E_{i}$. For any $t \in \mathbf{C}^{*}$ define a diagonal matrix $\lambda(t)$ whose $k$-th diagonal is $t^{m-i}$ if $r_{i-1}<k \leq r_{i}$. Then for any element $g \in P$ if we set $g(t)=\lambda(t) g \lambda(t)^{-1}$, the limit $g(0)=\lim _{t \rightarrow \infty} g(t)$ exists in $P$ and preserves the subspaces $V_{i}$ spanned by $e_{j}$ with $r_{i-1}<j \leq r_{i}$. Now suppose that $E$ is defined by a system of transition functions $\left\{g_{i j}\right\}$ with values in $P$ with respect to some open covering of $C$. Then it is clear that the family of systems of transition functions $\left\{g_{i j}(t)\right\}_{t \in \mathbf{C}}$ represents a family of vector bundles with the required property.

Lemma 5. Let $F_{i}, 1 \leq i \leq m$, be as in Lemma 3 , and set $F=\bigoplus_{i=1}^{m} F_{i}$. Then on $X=\mathbf{P}(F)$ a class $\gamma=-a \ell+b f, a, b \in \mathbf{R}$, is a Kähler class of $a>0$ and $b>a \mu\left(F_{1}\right)$.

Proof. As follows immediately from Lemma 2 we can find a hermitian metric $h_{i}$ on each $F_{\imath}$ so that the curvature form $\Theta$ of the resulting hermitian metric on $F$ takes the form

$$
(i / 2 \pi) \Theta=D\left(\mu\left(F_{1}\right) I_{1}, \cdots, \mu\left(F_{m}\right) I_{m}\right) \otimes \omega_{c},
$$

where $D\left(A_{1}, \cdots, A_{m}\right)$ denotes the diagonal matrix with entries $A_{\imath}$, and $I_{i}$ is the identity of $F_{\imath}$. By $\S 1$ (3) $\gamma$ is represented by the form

$$
\omega:=a \omega_{X / C}+\left(-a(i / 2 \pi) \bar{\Theta}+b q^{*} \omega_{C}\right) .
$$

Take any point $\xi \in F-C$ with $h(\xi, \xi)=1$. Write $\xi=\sum_{i=1}^{m} \xi_{i}$ with $\xi_{i} \in F_{\imath}$. Then by the definition of $\bar{\Theta}$ and (6) we get

$$
-a(i / 2 \pi) \bar{\Theta}_{\bar{\xi}}+b q^{*} \omega_{C}=-a\left(\sum_{i=1}^{m} \mu\left(F_{i}\right) h\left(\xi_{i}, \xi_{i}\right)\right) q^{*} \omega_{C},
$$

where $\bar{\xi} \in X$ is the image of $\xi$. Since $a>0$ and $\mu\left(F_{1}\right) \geq \mu\left(F_{i}\right)$, we have

$$
-a\left(\sum_{i=1}^{m} \mu\left(F_{i}\right) h\left(\xi_{i}, \xi_{i}\right)\right)+b \geq-a \mu\left(F_{1}\right)+b>0 .
$$

Thus the second term of $\omega$ is positive on the horizontal subbundle $H$. Together with the property of $\omega_{X / C}$ mentioned in Section 1 this implies that $\omega$ is a Kähler form.

Let $E$ be a vector bundle of rank $r$ and $F$ a subbundle of rank $\nu$. Identifying the subbundle $Q:=\mathbf{P}(F) \subseteq X:=\mathbf{P}(F)$ with its class in $H^{2(r-\nu)}(X, \mathbf{R})$ we have 


$$
Q=e^{r-\nu}+n_{\nu} e^{r-\nu-1} f
$$

LeMma 6. We have $t(F):=\mu(F)-\mu(E)=-n_{\nu} / \nu$.

Proof. Let $c=-\ell$. Then in view of $\S 1(2)$ and (7) we may write $Q=c^{r-\nu}$ $+n_{\nu}^{\prime} c^{r-\nu-1} f$ for a unique integer $n_{\nu}^{\prime}$ such that $s:=(r-\nu) c^{r}+r n_{\nu}^{\prime}=r n_{\nu}$. Hence it suffices to show that $t(F)=-s / r \nu$. In fact, we have

$$
-c_{1}(F)=Q c^{\nu}=\left(e^{r-\nu}+n_{\nu}^{\prime} c^{r-\nu-1} f\right) c^{\nu}=-c_{1}(E)+n_{\nu}^{\prime}
$$

so that $s=(r-\nu)\left(-c_{1}(E)\right)+r n_{\nu}^{\prime}=-r \nu t(F)$ as desired.

Proof of Proposition 1. Necessity. Suppose that $\gamma$ is a Kähler class. Then we have $0<r^{r}=(a e+b f)^{r}=r a^{r-1} b$, and for the class $u$ of a line in a fiber of $q$ we get $0<(a e+b f) \cdot u=a$. Hence we have $a, b>0$. Moreover, with $F$ and $Q$ as before we have $0<Q \gamma^{\nu}=a^{\nu-1}\left(a n_{\nu}+b \nu\right)$. Hence, by Lemma $6 b>-\left(n_{\nu} / \nu\right) a$ $=t(F) a$. Since $F$ is arbitrary, we get $b>t(X) a$.

Sufficiency. If $X$ is stable and $a, b>0, \gamma_{a b}$ contains a Kähler form $\omega_{a b}$ of $\S 1$ (4). So we may assume that $X=\mathbf{P}(E)$ for some non-stable bundle $E$ (cf. Remark 1). We consider a filtration $0=E_{0} \subseteq \cdots \subseteq E_{m}=E$ of $E$ as in Lemma 2. By Lemma 4 we can find a holomorphic family $\left\{X_{t}\right\}_{t \in \mathbf{C}}$ of ruled manifolds such that $X_{t} \cong X$ for $t \neq 0$ and $X_{0} \cong \mathbf{P}\left(\bigoplus_{i=1}^{m}\left(F_{i}\right)\right)$, where $F_{i}=E_{i} / E_{i-1}$. If $\gamma=\gamma_{a b}$ is a Kähler class on $X_{0}$ (considered naturally as an element of $H^{2}(X, \mathbf{R})$ ), then by continuity $\gamma$ is also a Kähler class on $X_{t}$ (considered naturally as an element of $H^{2}\left(X_{t}, \mathbf{R}\right)$ ) for all sufficiently small $t$ (cf. e.g. [7; Lemma 1]). Hence, by replacing $X$ by $X_{0}$ if necessary we may assume from the beginning that $E=\bigoplus_{i=1}^{m} F_{i}$. In this case $\gamma=-a \ell+(a \mu(E)+b) f($ cf. $\S 1(2))$ and it is a Kähler class if $a>0$ and $(a \mu(E)+b)>\mu\left(F_{1}\right)=a \mu_{\max }$, or equivalently, $a>0$ and $b>a t(X)$.

\section{§ 3. Generalized Kähler-Einstein metrics on ruled manifolds}

Let $X$ be a ruled manifold over $C$. For simplicity we assume that $p \geq 1$ in the sequel, where $p=p(C)$ is the genus of $C$. In this case we donote by $L(X)$ the identity component of the relative automorphism group Aut $X / C$ of $X$ over $C$. When $p \geq 2$, it coincides with the identity component $A_{u} t_{0} X$ of the automorphism group of $X$, while when $p=1$ the other case also occurs where the quotient group $T(X)=$ Aut $_{0} X / L(X)$ is isogenous to the complex torus Aut $C$. In all cases $L(X)$ is the maximal connected linear algebraic subgroup of $\mathrm{Aut}_{0} X$. We call $X$ simple if $X \cong \mathbf{P}(E)$ for a simple vector bundle $E$, where $E$ is by definition simple if the scalar multiplication is the only endomorphisms of $E$. Then the fol- 
lowing holds true (cf. the proof of Lemma 9, 2) below for the second statement).

Lemma 7. $L(X)$ reduces to the identity if and only if $X$ is simple. In this case any extremal Kähler metric is of constant scalar curvature.

Now let $g$ be a Kähler metric and $\pi:(\tilde{X}, \tilde{g}) \rightarrow(X, g)$ the universal covering of $(X, g)$. Then the metric $g$ is called a generalized Kähler-Einstein metric if the de Rham decomposition $(\tilde{X}, \tilde{g})$ is isomorphic to $\left(\widetilde{C}, g_{\tilde{C}}\right) \times\left(\mathbf{P}, g_{\mathbf{P}}\right)$, where $g_{\tilde{C}}$ and $g_{\mathbf{P}}$ are Kähler-Einstein metrics on $C$ and $\mathbf{P}$ respectively. Such a metric is of constant scalar curvature and hence is extremal in its Kähler class.

LeMma 8. Let $X$ be a ruled manifold with $p \geq 1$. If there exists a generalized Kähler-Einstein metric on $X$, then $X$ is quasistable. Conversely, if $X$ is quasistable, then for any Kähler class $\gamma_{a b}, a, b>0$, there exists a unique generalized Kähler-Einstein metric $g_{a b}$ in $\gamma_{a b}$ up to the natural action of $L(X)$.

Proof. The uniqueness assertion is a special case of the main result of [6], which also shows that if $(X, g)$ is generalized Kähler-Einstein, it can be written in the form $(X, g)=\left(\tilde{Y}, g_{\tilde{Y}}\right) \times\left(F, g_{F}\right) / \Gamma_{\rho}$ for some compact Kähler-Einstein manifolds $\left(Y, g_{Y}\right)$ and $\left(F, g_{F}\right)$ with seminegative and positive Ricci tensors respectively, where $\left(\tilde{Y}, g_{\tilde{Y}}\right)$ is the universal covering space of $\left(Y, g_{Y}\right)$, and $\Gamma_{\rho}=$ $\left\{(\gamma, \rho(\gamma)) ; \gamma \in \pi_{1}(Y)\right\}$ for some homomorphism $\rho: \pi_{1}(Y) \rightarrow \operatorname{Aut}\left(Y, g_{F}\right)$. In our case clearly we have $\widetilde{Y}=\widetilde{C}$ and $F=\mathbf{P}$; hence $X$ is quasistable. Conversely, if $X$ is quasistable, the Kähler metric $g_{a b}$ corresponding to the Kähler form $\omega_{a b}$ in $\S 1(5)$ gives the desired metric.

We call the extremal Kähler metrics $g_{a b}$ in the above lemma the canonical extremal (Kähler) metrics on $X$. Our basic questions in this note are then the following:

Question. Are the following statements true?

$1_{a b}$ : For a quasistable ruled manifold $X, g_{a b}$ is the unique extremal Kähler metric in the Kähler class $\gamma_{a b}, a, b>0$, up to the natural action of $L(X)$. (Note that by [4; Th. 4] any other extremal Kähler metric in $\gamma_{a b}$, if any, would also be of constant scalar curvature.)

2: A simple ruled manifold $X$ which is not quasistable admits no extremal Kähler metric (in any Kähler class).

In the next two sections we give examples of affirmative answers to these two questions in certain special cases. Also we show in Section 6 how these two problems are interrelated. 


\section{$\S 4$. Two special cases}

Let $\rho: \pi \rightarrow K$ be a homomorphism. In this section we shall prove the following:

Theorem 1. Let $X=X_{o}$ be a quasistable ruled manifold as in Lemma 2. Suppose that either 1) the image $\rho(\pi)$ is a finite subgroup of $K$, or 2) $C$ is an elliptic curve. Then Question $1_{a b}$ has an affirmative answer for any $a, b>0$.

First recall general results of Lichnerowicz and Calabi in terms of automorphism groups.

Lemma 9. 1) Let $X$ be a ruled manifold with a Kähler metric $g$ of constant scalar curvature. Then $L(X)$ is a reductive algebraic group and $\operatorname{Aut}(X, g) \cap L(X)$ is a maximal compact subgroup of it; furthermore the natural map $\operatorname{Aut}_{0}(X, g) \rightarrow T(X)$ is surjective. Here $\operatorname{Aut}(X, g)$ denotes the group of biholomorphic isometries and $\operatorname{Aut}_{0}(X$, g) its identity component.

2) If $L(X)$ is unipotent and is of positive dimension, then $X$ admits no extremal Kähler metric (in any Kähler class).

Proof. 1) Let $L_{a}$ be the complex Lie algebra of holomorphic vector fields on $X$ and $I$ (resp. $B$ ) the subalgebra of $L_{a}$ of those vector fields which are annihilated by any holomorphic 1 -form on $X$ (resp. which are parallel), where $B$ is in the center of $L_{a}$. Now the theorem in $[15 ; \S 10]$ states that $L_{a}$ is generated by the Lie algebra of $\operatorname{Aut}_{0}(X, g)$. Note that $I$ is identified with the Lie algebra of $L(X), B$ generates a subgroup of $\operatorname{Aut}_{0}(X, g)$, and the natural projection $L_{a} \rightarrow L_{a} / I \cong B$ corresponds to the homomorphism of Lie groups $\operatorname{Aut}_{0}(X, g) \rightarrow T(X)$ (cf. [5; Prop. 6.7]). The assertion follows from this.

2) Let $g$ be an extremal Kähler metric on $X$. In view of 1 ) we may assume that $g$ is not of constant scalar curvature. Then the vector field corresponding to the $(0,1)$-form $\bar{\partial} R_{g}$ is in $I$ and generates a nontrivial one parameter subgroup of $\operatorname{Aut}_{0}(X, g) \cap L(X)$, where $R_{g}$ is the scalar curvature of $g$ (cf. [15; §9][3; Th. 2.1]). Thus in this case also $L(X)$ is not unipotent.

In view of Lemma 8 the theorem follows from:

LMmea 10. Let $X_{\rho}$ be as in Theorem 1. Then any Kähler metric $g$ with constant scalar curvature on $X_{\rho}$ is a generalized Kähler-Einstein metric.

Proof. 1) Assume that $\rho(\pi)$ is a finite group. Then after passing to a suitable unramified covering we may assume that $X=C \times \mathbf{P} . L(X)$ is then naturally 
identified with $G:=$ Aut $\mathbf{P}$. Since $g$ is of constant scalar curvature, it is left fixed by a maximal compact subgroup $K_{g}$ of $G$ by Lemma 9 . By replacing $g$ by $h^{*} g$ for some $h \in G$ with $h K_{g} h^{-1}=K$ we may further assume that $K_{g}=K$. Let $p_{1}: X$ $\rightarrow C$ and $p_{2}: X \rightarrow \mathbf{P}$ be the natural projections. Then according to the Künneth decomposition of the bundle $\wedge^{1,1}$ of $(1,1)$-forms on $X$

$$
\wedge^{1,1}=p_{1}^{*} \wedge_{C}^{1,1} \oplus p_{2}^{*} \wedge_{\mathbf{P}}^{1.1} \oplus\left(p_{1}^{*} T_{C}^{*} \otimes p_{2}^{*} \bar{T}_{\mathbf{P}}\right) \oplus\left(p_{1}^{*} \bar{T}_{C}^{*} \otimes p_{2}^{*} T_{\mathbf{P}}^{*}\right)
$$

we have the decomposition

$$
\omega=\omega_{11}+\omega_{22}+\omega_{12}+\omega_{21}, \bar{\omega}_{12}=\omega_{21}
$$

of the Kähler form $\omega=\omega_{g}$ with each component again $K$-invariant. Fix any point $p \in C$ and a local parameter $z$ around $p$. Then on the fiber $\mathbf{P}(p):=p \times \mathbf{P}$ we may write $\omega_{21}=\phi d \bar{z}$ for a unique $K$-invariant $C^{\infty}(1,0)$-form $\phi$ on $\mathbf{P}(p) \cong \mathbf{P} ; \phi$ thus is $d$-closed and hence vanishes identically. We get: $\omega_{12}=\omega_{21}=0$. Further, we may write $\omega_{11}=p_{1}^{*} \omega_{1}$ for some Kähler form $\omega_{1}$ on $C$ and $\omega_{22}=p_{1}^{*} f \cdot p_{2}^{*} \omega_{\mathrm{P}}$ for some $C^{\infty}$ function $f$ on $C$. Then from $d \omega_{22}=0$, we deduce that $f$ is actually a constant. Since $\omega$ is of constant scalar curvature, the same must be true also for $\omega_{1}$; hence $\omega_{1}$ is also Kähler-Einstein. Thus the lemma is proved in this case.

2) Assume that $C$ is an elliptic curve. Then $c_{1}(X)=c_{1}(X / S)=r e$, and it is represented by the semipositive $(1,1)$-form $\omega_{r 0}$. Hence by $[16, \S \S 12,13]$ the natural homomorphism $\mathrm{Aut}_{0} X \rightarrow \mathrm{Aut}_{0} C$ is surjective, then since $g$ is of constant scalar curvature, by Lemma $9 \operatorname{Aut}_{0}(X, g)$ also is mapped surjectively to Aut $_{0} C$. Passing to the universal covering $\widetilde{X}=\mathbf{C} \times \mathbf{P}$ we see that $\operatorname{Aut}_{0}(X, g)$ is covered by a product group of the form $\mathbf{C} \times \operatorname{Aut}\left(\mathbf{P}, g_{\mathbf{P}}\right)$, where $g_{\mathbf{P}}$ is some Kähler metric on $\mathbf{P}$. Especially, the induced Kähler metric $\tilde{g}$ on $\widetilde{X}$ is invariant by the $\mathbf{C}$-action on the first factor. We now consider the Künneth decomposition as (8) for the Kähler form $\omega=\omega_{\tilde{g}}$ on $\mathbf{C} \times \mathbf{P}$; for example $\omega_{11}=\omega_{11}(\zeta)$ (resp. $\left.\omega_{22}=\omega_{22}(t)\right)$ is a $C^{\infty}$ family of $(1,1)$-forms on $\mathbf{C}$ (resp. $\mathbf{P}$ ) parametrized by $\mathbf{P}$ (resp. $\mathbf{C}$ ). By the $\mathbf{C}$-invariance $\omega_{22}$ is $d$-closed. If we write $\omega_{21}=\phi \wedge d \bar{t}$ for some $C^{\infty}$ family $\phi=\phi(t)$ of $C^{\infty}(1,0)$-forms on $\mathbf{P}$ parametrized by $t \in \mathbf{C}$, this implies that $\bar{\partial}(\phi(t))=0$ on $\mathbf{P}(t):=t \times \mathbf{P}$; hence $\phi=0$ and $\omega_{12}=\omega_{21}=0$. This in turn implies that $\omega_{11}$ is $d$-closed, and hence is independent of $\zeta \in \mathbf{P}$. Thus we have $\omega=$ $p_{\mathbf{C}}^{*} \omega_{1}+p_{\mathbf{P}}^{*} \omega_{2}$ for some Kähler forms $\omega_{1}$ on $\mathbf{C}$ and $\omega_{2}$ on $\mathbf{P}$. Since $\omega$ is of constant scalar curvature, so are $\omega_{1}$ and $\omega_{2}$. The lemma follows.

In the case of ruled surfaces over an elliptic curve the general situation is as follows.

EXAmple. Any ruled surface of genus 1 is isomorphic to one of the following 
ones (cf. [20]): a) $X_{n}=\mathbf{P}\left(1_{C} \oplus L\right), \operatorname{deg} L=n>0$. b) $X_{L}=\mathbf{P}\left(1_{C} \oplus L\right)$, $\operatorname{deg} L=0$, c) $X_{0}=\mathbf{P}\left(F_{0}\right)$, and d) $X_{M}=\mathbf{P}\left(F_{M}\right)$, deg $M=1$, where $F_{0}$ (resp. $\left.F_{M}\right)$ is the unique vector bundle obtained as a nontrivial extension of $1_{C}$ (resp. $M$ ) by $1_{C}$. Here $L$ and $M$ denote line bundles over $C$ in general and $1_{C}$ denotes the trivial line bundle. Among these, only $X_{n}$ and $X_{0}$ are not quasistable. Since $L(X)$ of these surfaces are not reductive (cf. [17; Th. 2(3)]), they never admit a Kähler metric with constant scalar curvature.

Remark 2. Similarly, in general no nonsimple semistable ruled surfaces admit an extremal Kähler metric. In fact, such a ruled surface is written as $X=\mathbf{P}(E)$, where $E$ is obtained as a nontrivial extension

$$
0 \rightarrow 1_{C} \rightarrow E \rightarrow 1_{C} \rightarrow 0,
$$

and we have $L(X) \cong \mathbf{C}$ naturally (cf. [17; Th. 2(2)]).

\section{§ 5. Case with vanishing curvature}

In this section we assume that $X$ is a ruled surface. We shall make precise the result of Burns and de Baltolomeis [1]. Denote by $E_{K}$ the unique vector bundle of rank 2 which is obtained as the nontrivial extension $0 \rightarrow K_{C} \rightarrow E_{K} \rightarrow 1_{C} \rightarrow 0$. $E_{K}$ is not stable. Let $p$ be the genus of $C$.

LEMma 11. 1) If a class $\gamma$ in $H^{2}(X, \mathbf{R})$ is a class containing a Kähler metric $g$ with vanishing scalar curvature, then $\gamma$ is of the form $c((e+(p-1) f))=$ $(1 / 2) c c_{1}\left(K_{X / C}^{-1} \otimes q^{*} K_{C}\right)$ for some real number $c>0$. Any Kähler metric with constant scalar curvature in such a class has vanishing scalar curvature.

2) A class $\gamma$ of the form $\gamma=c(e+(p-1) f)$ with $c>0$ is a Kähler class on $X$ if and only if $p \geq 2$ and $X \supsetneqq \mathbf{P}\left(E_{K}\right), \mathbf{P}\left(1_{C} \oplus L\right)$, where $L$ is a line bundle with $\operatorname{deg} L \leq 2(1-p)$.

Proof. 1) If $R_{\boldsymbol{g}}$ is the scalar curvature of $g$, we have

$$
2 \pi c_{1}(X) \cdot \gamma=\int_{X} R_{g} d v_{g} / \int_{X} d v_{g},
$$

where $d v_{g}$ is the volume form of $g$. The assertions then follow from the relation $c_{1}(X)=(2-2 p) f+2 e$.

2) Let $s=s(X)$ be the minimum of the self-intersection number of the sections of $X$. Then we have $t(X)=-s / 2$. Hence, by Proposition $1 \gamma$ is a Kähler class if and only if $2 p>2-\min (0, s)$, i.e., if and only if $p \geqq 2$ and $2(1-p)<s$. On the other hand, if $X$ is indecomposable, we have $s \geq 2(1-p)$ and the equality holds if and only if $X$ is isomorphic to $\mathbf{P}\left(E_{K}\right)$ (cf. Th. 2.12 and 
its proof in [12; V]). If $X$ is decomposable, we may write $X=\mathbf{P}\left(1_{\mathrm{c}} \oplus L\right)$ for some line bundle $L$ with $\operatorname{deg} L \leq 0$. Then we have $s=\operatorname{deg} L$ (cf. [12; V, 2.11.2, 3]). The lemma follows from these.

The following result is essentially due to Burns and de Baltolomeis [1].

Proposition 2. On a ruled surface $X$ any Kähler metric $g$ with vanishing scalar curvature is a generalized Kähler-Einstein metric.

The proof is the same as that of Lemma 2 in [1] though it is proved there for a certain special ruled surface; in fact the proof shows that $g$ is locally symmetric, and hence in particular a generalized Kähler-Einstein metric. Combined with Lemmas 11 and 2 this yields:

TheORem 2. Let $X$ be a ruled surface satisfying the last condition of Lemma 12. Then Question $1_{a b}$ and Question $2(\S 3)$ have the affirmative answers for Kähler classes of the form $\gamma=c(e+(p-1) f), c>0$. (Thus $(a, b)=(c, c(p-1))$.)

Remark 3. If $X$ is a compact connected Kähler surface admitting a Kähler metric $g$ with vanishing scalar curvature, then either $c_{1}(X)=0$ or $X$ is birationally ruled ([21; Th. 2]).

\section{\$ 6 Interrelation of the two questions}

Let $a$ and $b$ be positive real numbers. In this section we assume for simplicity that the base curve $C$ of a ruled manifold $X$ has genus $p(C) \geq 2$. By a standard argument (cf. the proof of [19; Prop. 7.5]) one gets immediately the following:

Lemma 12. Let $\left\{X_{t}\right\}$ be a holomorphic family of stable ruled manifolds parametrized by a complex manifold $T$. Denote by $g_{a b}(t)$ the canonical extremal metric $g_{a b} d e$ fined on $X=X_{t}$. Then $g_{a b}(t)$ depends smoothly on $t$.

Lemma 13. Let $\left\{X_{t}\right\}$ be a holomorphic family of ruled manifolds parametrized by the unit disc $D=\{t \in C ;|t|<1\}$ such that $X_{t}$ is stable for any $t \neq 0$. Let $\left\{g_{t}\right\}_{t \in D}$, be the $C^{\infty}$ family of extremal metrics $g_{t}=g_{a b}(t)$ on $X_{t}$ over $D^{\prime}=D-0$. If this family extends to a $C^{\infty}$ family of Kähler metrics $\left\{g_{t}\right\}_{t \in D}$ across 0 , then $X_{0}$ is quasistable.

Proof. Suppose that an extension $\left\{g_{t}\right\}_{t \in D}$ as above exists. Let $T_{X_{t}}$ be the holomorphic tangent bundle of $X_{t}$. Let $H_{t}$ be the orthogonal complement of the vertical tangent bundle $T_{X_{t} / C}$ in $T_{X_{t}}$ with respect to $g_{t} . H_{t}$ is then a $C^{\infty}$ complex line subbundle of $T_{X t}$ which is holomorphic for $t \neq 0$. We show that $H_{0}$ also is holomorphic. In fact, $H_{t}$ defines a $C^{\infty}$ section $\sigma_{t}: X_{t} \rightarrow \mathbf{P}_{t}$ of the natural projection $\mathbf{P}_{t}:=$ 
$\mathbf{P}\left(T_{X_{t}}\right) \rightarrow X_{t}$, which is holomorphic if $t \neq 0$. Then we may consider $\bar{\partial}_{t} \sigma_{t}$ for any $t$ with respect to local coordinates. Since it vanishes for $t \neq 0$, so does it for $t=0$ by continuity. Thus $H_{0}$ is a holomorphic subbundle and we get the orthogonal holomorphic decomposition $T_{X_{0}}=T_{X_{0} / C} \oplus H_{0}$. Moreover, as a limit of integrable distributions $H_{t}, H_{0}$ also is integrable. This implies that $\left(X_{0}, g_{0}\right)$ is locally a product of two Kähler submanifolds arising from the foliations defined by $T_{X_{0} / C}$ and $H_{0}$. Since $g_{0}$ is of constant curvature as well as $g_{t}$, the same must also be true for both of the two factors; hence $\left(X_{0}, g_{0}\right)$ is generalized Kähler-Einstein. $X_{0}$ is then quasistable by Lemma 8 .

Theorem 3. Let $X$ be a simple non-quasistable ruled manifold. If there exists an extremal Kähler metric on $X$ in a class $\gamma_{a b}$, then for any stable ruled manifold $X^{\prime}$ which is a sufficiently small deformation of $X$ the class $\gamma_{a b}$ contains an extremal Kähler metric other than the canonical one $g_{a b}$.

In particular, if Question $1_{a b}$ has an affirmative answer in general, Question 2 has also the affirmative answer.

Lemma 14. Let $X$ be a simple ruled manifold. Let $f: \mathscr{X} \rightarrow S, X_{0}=X, o \in S$, be a deformation of $X$. Then for any extremal Kähler metric $g$ on $X$ in a Kähler class $\gamma_{a b}$ there exists a unique $C^{\infty}$ family $\left\{g_{s}\right\}_{s \in S}$ of extremal Kähler metrics $g_{s}$ on $X_{s}:=$ $f^{-1}(s)$ in $\gamma_{a b}$ (after restricting $S$ around $o$ ).

Proof. Clearly, $\tilde{\gamma}:=\left\{\gamma_{a b}(s)\right\}_{s \in S}$ forms a section of the locally constant sheaf of $R^{2} f_{*} \mathbf{R}$ and $\gamma_{a b}(s)$ is of type $(1,1)$ on each fiber. In view of Lemma 7 the result then follows from [8; Th. 6.3.].

Proof of Theorem 3. Let $g$ be any extremal Kähler metric on $X$ in $\gamma_{a b}$. Let $f: \mathscr{X} \rightarrow S, X_{0}=X, o \in S$, be a Kuranishi family of $X$, where $S$ is nonsingular (cf. [19; Th. 4.2]). By Lemma $14 g$ extends to a unique $C^{\infty}$ family $\left\{g_{s}\right\}_{s \in ́}$ of extremal Kähler metrics $g_{s}$ on $X_{s}$, if we restrict $S$ around $o$. Let $U=\left\{s \in S ; X_{s}\right.$ is stable\}. Then $U$ is a dense Zariski open subset of $S$ (cf. [19; Prop. 4.1]). It then suffices to show that for any $s \in U, g_{s} \neq g_{a b}$; indeed, if $g_{s}=g_{a b}$ for some point $s$ $\in U$, then again by Lemmas 12 and 14 we must have $g_{a b}=g_{s}$ for all $s \in U$; then the restriction of $f$ to any unit disc $D$ embedded in $S$ with $D \cap(S-U)=\{o\}$ gives rise to a contradiction to Lemma 13 .

\section{REFERENCES}

[1] Burns, D. and de Baltolomeis P., Stability of vector bundles and extremal metrics, Invent, math., 92 (1988), 403-408. 
[2] Bando, S and Mabuchi, T., Uniqueness of Einstein-Kähler metrics modulo connected group actions, In: Oda, T. (ed.) Algebraic geometry, Proceeding, Sendai 1985 (Advanced Studies in Pure Math., 10, pp. 11-40): Kinokuniya and North-Holland 1986.

[3] Calabi, E., Extremal Kähler metrics, (Seminar on differential geometry, S.-T. Yau ed., Princeton 1979), Ann, of Math. Studies, 102 (1982), 259-290.

[4] - Extremal Kähler metrics II, In: Differential geometry and complex analysis dedicated to E. Rauch (Cheval, I. and Farkas, H. M. eds.) Springer-Verlag, 1985, 95-114.

[5] Fujiki, A., On automorphism groups of compact Kähler manifolds, Invent. Math., 44 (1978), 225-258.

[6] - On the uniqueness of generalized Kähler-Einstein metric, Math. Göttigenesis, Heft 46, 1988.

[ 7 ] - Coarse moduli spaces for polarized compact Kähler manifolds, Publ. RIMS, Kyoto Univ., 20 (1984), 977-1005.

[8] Fujiki, A. and Schumacher G., On the moduli space of extremal compact Kähler manifolds, Publ. RIMS, Kyoto Univ., 26 (1990), 101-183.

[9] Futaki, A., On compact Kähler manifolds of constant scalar curvature, Proc. Japan Acad., 59 (1983), 401-402.

[10] Griffiths, P. A., Hermitian differential geometry, Chern classes, and positive vector bundles, In: Iyanaga, S., Spencer, D. C., (eds.) Global Analysis, Princeton Univ. Press, 1969.

[11] Harder, G., and Narasimhan, M. S., On the cohomology groups of the moduli spaces of vector bundles on curves, Math. Ann., 211 (1975), 215-248.

[12] Hartshorne, R., Algebraic geometry, Graduates Texts in Math., 52, BerlinHeidelberg-New York: Springer, 1977.

[13] Lange, H., Zur Klassifikation von Regelmannigfaltigkeiten, Math. Ann., 262 (1983), $447-459$.

[14] Levine, M., A remark extremal Kähler metrics, J. Diff. Geom, 21 (1985), 73-77.

[15] Lichnerowicz, A., Osométries et transformations analytiques d'une variété kähérienne commpacte, Bull. Soc. Math. France, 87 (1959), 427-437.

[16] Lichnerowicz, A., Variétés kählériennes à premiére classe de Chern non-négative et variétés riemanniennes à courbure de Ricci généralisée non-négative, J. Diff. Geom., 6 (1971), 47-94.

[17] Maruyama, M., On automorphism groups of ruled surfaces, J. Math. Kyoto Univ., 11 (1971), 89-112.

[18] Narasimhan, M. S., and Seshadri, C. S., Stable and unitary vector bundles on a compact Riemann surface, Ann. of Math., 82 (1965), 540-567.

[19] Ramanathan, A., Stable principal bundles on a compact Riemann surface, Math. Ann., 213 (1975), 129-152.

[20] Suwa, T., Ruled surfaces of genus one, J. Math. Soc. Japan, 2 (1969), 258-291.

[21] Yau, S. T. On the curvature of compact Hermitian manifolds, Invent. Math, 25 (1974), 213-239.

Kyoto University

Yoshida Collge

Kyoto 606, Japan 